\title{
Mein Großvater, die Unitarian Church und ich: Persönliche Anmerkungen
}

\author{
Frido Mann
}

Ende Juli 2010 bekam ich von Heinrich Detering ein Vortragsmanuskript zugeschickt. Es war überschrieben Thomas Mann und die Unitarier und sollte während der Davoser Literaturtage im August vorgestellt werden. Der Vortrag begann mit Deterings Hinweis, dass er dieses bisher unbemerkt gebliebene Thema der ThomasMann-Forschung ,abseits der großen Scheinwerferkegel“ habe aufgreifen wollen. Immerhin war ja - aber das hatte bislang niemand so recht beachtet - schon in der Entstehung des Doktor Faustus unmissverständlich nachzulesen, wie Thomas Mann den amerikanischen Unitariern dankte für ,die angenehmste kirchliche Erfahrung, die ich gemacht habe"; und auch sein Brief an einen unitarischen Pastor wenige Monate vor seinem Tod lag längst gedruckt vor: „Der Geist Ihrer Kirche [...] dieser Geist ist es, der mich anzieht, seit ich ihn kennen lernte“.

Heinrich Detering hatte fünf Jahre zuvor bei der Lübecker Tagung zu Thomas Manns fünfzigstem Todestag meinen Vortrag Thomas Mann und die Frage der Religion gehört. Dort hatte ich insbesondere auf Thomas Manns Verhältnis zur Unitarischen Kirche in Los Angeles während seines kalifornischen Exils aufmerksam gemacht und überraschenderweise nur sehr zurückhaltende Resonanz gefunden. Meine noch sehr thesenhaften Hinweise hatten sich wesentlich der Lektüre eines Aufsatzes des katholischen Theologen Hans Küng verdankt: Thomas Mann und die Frage der Religion (erschienen in dem mit Walter Jens herausgegebenen Band Anwälte der Humanität). Dort wurde nicht nur betont, dass das Religiöse in Thomas Manns Werk seit den Joseph-Romanen immer breiteren Raum einnahm, sondern es wurde 
auch dessen Beziehung zum amerikanischen Unitarismus erwähnt, einschließlich der unitarischen Taufe aller vier Enkel auf Veranlassung Thomas Manns. Dessen Hinwendung zur Unitarischen Kirche kurz vor der Entstebung des Doktor Faustus mit seinen im Vergleich zur Joseph-Tetralogie deutlich elementareren, religiösen Untertönen interpretierte ich daraufhin in meinem Lübecker Vortrag als eine unter dem Eindruck von kalifornischer Exil-Ferne, Heimatlosigkeit, Schock des Faschismus und des Krieges stehende Suche nach einer geistlichen Heimat im Schutzraum des amerikanischen Exils.

Mein Wunsch, diese neue Sicht anlässlich Thomas Manns rundem Todestag vorzutragen, war nicht primär wissenschaftlichem Interesse, sondern persönlicher Bewegtheit entsprungen: Hier taten sich für mich neue biografische Zusammenhänge auf zwischen Thomas Manns Beziehung zur amerikanischen Kirche und meiner eigenen Taufe. Ich konnte dies alles nun neu als Hintergrund auch für meine persönliche religiöse Entwicklung verstehen, einschließlich meiner Konversion zum Katholizismus und des nachfolgenden Theologiestudiums. Immerhin hatte ich bis wenige Jahre zuvor nie etwas von Thomas Manns Verhältnis zu dieser amerikanischen Kirche gewusst. Hatten doch weder mein Großvater noch irgendwelche anderen Familienmitglieder, ja nicht einmal meine Eltern während meiner ganzen Kindheit und Jugend je mit mir über all dies gesprochen. Religion gehörte in der Mann-Familie offenbar zu den vielen intimen Tabuthemen: darüber sprach man einfach nicht.

Nun aber las ich in Deterings Text von Thomas Manns Hinwendung zur von der amerikanischen Aufklärung geprägten Unitarischen Kirche, seinem Bemühen um eine Verschmelzung der Traditionen seiner europäischen Herkunft mit der amerikanischen Kultur, las neue Dokumente über die enge Beziehung Thomas Manns zur Unitarischen Kirche in Los Angeles von den frühen vierziger Jahren bis Ende 1954, seine unitarische Kanzelrede von 1951 ebenso wie die bisher teilweise unveröffentlichte Korrespondenz zwischen Thomas Mann und zwei unitarischen Pastoren. Zum einen war da Stephen Fritchman, der, ursprünglich Methodistenpfarrer, 1948 in Los Angeles die Leitung der Unitarischen Kirche von Los Angeles übernahm und bald im Zuge der „Kommunisten“-Hysterie McCarthys sich - mit Thomas Manns solidarischer Unterstützung! - gegen seine Denunziation zu wehren hatte und der unermüdlich für Toleranz und für eine Gleichberechtigung aller Rassen eintrat. Fritchman legte immer Wert auf seinen Titel Reverend, verstand sich jedoch nur in einem sehr weitläufigen Sinn als Christ und griff dabei auch entschieden ins Politische, Marxistische aus. Entdeckt hatte Detering auch jene ergreifende Trauerrede, die Fritchman zu Thomas Manns Tod 1955 vor seiner Gemeinde gehalten hatte.

Als völlig neue Figur aber wurde in Deterings Recherchen Fritchmans Vorgänger sichtbar: Ernest Caldecott. Und dieser Mann ging mich an. Denn Caldecott taufte, auf Thomas Manns Veranlassung, im Frühjahr 1942 dessen älteste Enkel Angelica - und mich. Diese enge und, vor allem, gegenseitige Beziehung zwischen diesem deutschen Schriftsteller und dieser amerikanischen Kirche warf ein neues 
Licht auf Thomas Manns Verständnis von Religion in seinen von religiös humanistischem und politischem Denken bestimmten späten Romanen und Essays.

Angesichts der sehr persönlichen Motive für meinen Lübecker Vortrag hatte ich seinerzeit auf wissenschaftliche Recherchen verzichtet; und ich hatte das Thema auch nach dem Lübecker Auftritt auf sich beruhen lassen. Umso überraschter war ich, als ich knapp drei Jahre später, kurz nach dem Erscheinen meiner (ebenfalls kurz auf den Unitarismus Bezug nehmenden) Autobiografie Acbterbabn, von der Schweizerischen Thomas-Mann-Gesellschaft Ende Mai 2008 zu einer Tagung mit dem neuartigen, aber vorsichtig im Plural formulierten Thema: „Thomas Mann und die Religionen" eingeladen wurde. In meinem Schlusswort griff ich noch einmal auf meinen Lübecker Vortrag zurück. Rund ein Jahr später folgte die mehrmonatige Vorlesungsreihe „Der ungläubige Thomas“ in Zürichs protestantischem Fraumünster, in Kooperation mit dem Großmünster. Und wiederum ein halbes Jahr später dann trug Heinrich Detering bei den Davoser Literaturtagen 2010 (mit dem allgemeinen Thema „Zwischen Himmel und Hölle. Thomas Mann und die Religion“, jetzt im Singular!) ebenjene Entdeckungen und Interpretationen vor, deren Manuskript er mir kurz zuvor zugeschickt hatte. Und dies stellte nun meine Überlegungen auf eine neue Grundlage. Sein aufsehenerregender Davoser Vortrag eröffnete neue Dimensionen für die Thomas-Mann-Forschung - und für mich. Er führte uns beide zum erstenmal persönlich zusammen, und ich wurde bei der Konzeption eines Buches über Thomas Manns amerikanische Religion zu seinem Gesprächspartner.

Wir trafen uns seitdem regelmäßig und sprachen über dieses Vorhaben, über Thomas Manns Verhältnis zur Religion, über meine Familie und über meine eigene religiöse Sozialisation. Heinrich Detering berichtete von seinen Funden in europäischen und amerikanischen Archiven, und ich konnte ihm im Gegenzug auto- und familienbiografische Hintergründe zeigen. Im Februar 2011 trug er dann, auf Einladung der Deutschen Forschungsgemeinschaft, in der Münchner Staatsbibliothek eine Kurzfassung seines schon weit fortgeschrittenen Buches vor. Zur Klärung und Schärfung seines Arguments trug bei, dass er sich in der Diskussion mit der Behauptung auseinanderzusetzen hatte, Thomas Manns Verhältnis zum amerikanischen Unitarismus sei doch nicht mehr gewesen als eine kurze Episode im Leben eines eingefleischten Lutheraners, zumal es sich bei jenen Unitariern ohnehin eher um eine politische als eine religiöse Gemeinschaft gehandelt habe. Dass freilich Thomas Manns Verhältnis zu den Unitariern eben als ein humanitäres und politisches für ihn gerade tiefgreifend religiös bestimmt war: das kristallisierte sich fortan in unseren fortgesetzten Diskussionen als die aus meiner Sicht zentrale These des Buches heraus.

Meine Taufe in der First Unitarian Church in Los Angeles 1942 hatte ich jahrzehntelang als ganz unabhängig von Thomas Mann betrachtet. Während meiner Kindheit und Jugend hatten weder meine Eltern noch irgendwelche anderen Verwandten je mit mir über die Unitarische Kirche, ja überhaupt nicht über Kirche, Religion oder Bibel gesprochen. Die ersten Anregungen, auf die ich mit umso 
größerer Neugierde, vielleicht auch mit ein bisschen Nachholbedarf reagierte, kamen erst nach meiner Übersiedlung nach Europa in meinem neunten Lebensjahr, und sie kamen nicht aus meiner Familie, sondern von außerhalb. So verfolgte ich vor allem aufmerksam den regelmäßigen sonntäglichen Kirchgang der streng katholischen Hausangestellten meiner Schweizer Großeltern bei Zürich, bei denen ich ja ein Jahr lang wohnte und zur Schule ging. Auch fragte ich diese Hausangestellte während unserer häufigen Gespräche in der Küche immer wieder nach religiösen Dingen aus. Mindestens einmal begleitete ich sie zur Sonntagsmesse in ihrer von zuhause zu Fuß ziemlich weit entfernten römisch-katholischen Kirche und kam von diesen Gottesdienstbesuchen beeindruckt zurück.

Auch in Österreich, wo ich danach mit meinen Eltern für zweieinhalb Jahre wohnte, bekam ich viel vom kirchlichen Landleben mit. Ich ließ mich von Nachbarkindern oder Mitschülern ebenfalls zu Gottesdiensten mitnehmen und nahm auch an der Beerdigung einer Großmutter in der Nachbarschaft teil, die ich in ihrem Zimmer zwischen Kerzen aufgebahrt gesehen hatte. Unter all diesen Eindrücken keimte in mir zum ersten Mal der Wunsch nach einer Konversion in die katholische Kirche auf. Als ich meine Mutter „um Erlaubnis“ bat zu konvertieren, meinte sie verständlicherweise, ich sollte diesen gewichtigen Schritt nicht übereilen, sondern noch zwei Jahre warten; danach könnte man diesen Wunsch ja immer noch erfüllen. Meine religiösen Anmutungen hielten tatsächlich nicht lange vor. Fünf Jahre später aber, nicht lange nach dem Tod meines Großvaters, flammte in dem Siebzehnjährigen dieser Gedanke während meiner Zeit bei meiner Großmutter Katia im Kilchberger Haus nochmals kurz auf. Bis zu meiner wirklich vollzogenen Konversion im Alter von dreiundzwanzig Jahren verging dann nochmals so viel Zeit.

Mein religiöses Urerlebnis im Zusammenhang mit einer Einstudierung von Wagners Bühnenweihfestspiel Parsifal während der vorösterlichen Zeit im Zürcher Opernhaus habe ich in meiner Autobiografie Achterbabn geschildert. Darüber hinaus war jedoch mein religiöses Wissen, auf dem Hintergrund einer völlig areligiösen Erziehung, immer noch gleich Null. Meine erste Informationsquelle zu „Religion“, „Christentum“ oder "Jesus Christus" war die Brockhaus-Enzyklopädie. Nach anfänglichen Recherchen tastete ich mich aus eigenen Stücken notdürftig weiter vor, bis schließlich hin zum Konvertitenunterricht bei einem älteren Jesuitenpater in Zürich, einem mit C. G. Jung befreundeten Theologen und Tiefenpsychologen. Dieser versah mich als erstes mit einem klassischen Katechismus und einer katholischen Ausgabe des Neuen Testaments. Monatelang verheimlichte ich allen meinen Verwandten, auch meiner Großmutter Katia, bei der ich lebte, diese seelsorgerlich angeleitete, intensive Vorbereitung auf meine geplante römischkatholische Taufe.

Denn diese Taufe war, wie sich nun herausstellte, eine Voraussetzung für meinen Eintritt in die katholische Kirche: Die unitarische Taufhandlung wurde dort, wie ich jetzt durch Heinrich Detering gelernt habe, genauso wenig anerkannt wie beim protestantischen Dachverband des Federal Council of Churches of America, die die 
Unitarische Kirche von der Mitgliedschaft ausschloss. Umso überraschter, ja fast bestürzt reagierte meine Großmutter, als sie mich, relativ kurz vor meinem Studienwechsel nach Rom und meiner dort vorgesehenen Taufe, einmal beim Studium des Neuen Testaments „erwischte“. „Sowas liest du?“, fragte sie mit großen Augen, um dann zu erklären, dass ihr diese Welt sehr fremd sei, obwohl es durchaus einige im Freundeskreis der Familie gäbe, die sich ,intensiv mit diesen Dingen beschäftigten“. Meinen etwa acht Jahre zuvor verstorbenen Großvater erwähnte sie in diesem Zusammenhang mit keinem Wort. Aber es beeindruckte sie sichtlich, dass ich mich so selbstständig „mit diesen Dingen“ auseinandersetzte.

Es wäre ein Leichtes gewesen, mir während der Hinwendung zum Religiösen über Konversion und Taufe mit Anfang Zwanzig aus dem Werk Thomas Manns ein Bild über dessen Verhältnis zur Religion zu machen. Aber genau darauf verzichtete ich, weil ich meine Konversion auch als eine Flucht aus der Familie empfand, hinein in die geistliche Weltgemeinde der römisch-katholischen Kirche. Maßgeblich für meine Entscheidung war der neue Aufbruch dieser Kirche mit der ökumenischen Bewegung und dem aggiomamento des Zweiten Vatikanischen Konzils unter Papst Johannes XXIII. während der frühen Sechziger Jahre gewesen. Als ich Jahrzehnte später dann die Werke Thomas Manns las, oft erst jetzt zum ersten Mal, da interessierten mich die religiösen Aspekte dieser Werke nicht mehr. Denn am Ende meines Theologiestudiums hatte ich mich in meiner Enttäuschung über die weitere Entwicklung der katholischen Kirche so weit von ihr entfernt, dass dies alles kein wichtiges Thema mehr für mich war. Meine Arbeit später als Psychologe in einem psychiatrischen Krankenhaus mit psychisch Schwerkranken und dann mit krebskranken Kindern auf einer onkologischen Klinikstation interpretierte ich nachträglich als einen sich wie ein roter Faden die Jahrzehnte durchziehenden Ausdruck einer nicht mehr religiösen, sondern nun entschieden humanistischen Einstellung, auch wenn ich bei dieser Arbeit oft sehr bewusst mit Extremsituationen an der Grenze zu den Letzten Dingen konfrontiert wurde.

Auch dass ich als Vierzigjähriger in Klaus Manns Der Wendepunkt nachlesen konnte, dass mein Onkel während seiner Zeit in der amerikanischen Armee im Krieg in Gesprächen mit dem katholischen Feldgeistlichen ernsthaft, aber nicht nachhaltig erwogen hatte, in die katholische Kirche überzutreten, empfand ich allenfalls als eine interessante Parallele zu meinem eigenen Weg. Noch weniger erkannte ich weitere gut zehn Jahre später die Zusammenhänge zwischen dem (mir damals halb verborgen gebliebenen) Eintritt meines Sohnes Stefan bei den Quäkern - im selben Alter, in dem ich in die katholische Kirche eingetreten war! - und dem gemeinsamen, wie sich herausstellte: auch im Unitarismus wurzelnden ,geistlichen Erbe“ unseres Groß- bzw. Urgroßvaters. Dies änderte sich, nachdem ich bereits um die Jahrtausendwende einen Weg zurück zum religiösen bzw. zu einem neuen religionsübergreifenden Denken gefunden hatte und ich mir über Hans Küngs erwähnten Aufsatz erste Einblicke in Thomas Manns Verhältnis zur Religion im Allgemeinen und zum Unitarismus im Besonderen verschaffen konnte. Ent- 
schieden bestätigt fand ich die Parallelen zwischen den Generationen gerade in der Frage der Religion in Heinrich Deterings Manuskript.

Jetzt standen mir die komplexen Zusammenhänge endlich klar vor Augen: die Zusammenhänge zwischen Thomas Manns später vergessener oder verschwiegener Verbindung zum Unitarismus, Klaus Manns abgebrochenem Vorstoß zu einer Konversion, meiner eigenen unitarischen Taufe und Hinwendung zu Religion und Kirche und dem Eintritt meines Sohnes Stefan bei den Quäkern. Gleichzeitig aber wirft Deterings Buch auch noch einmal die Frage auf, inwiefern Thomas Manns Hinwendung zu der betont humanistisch diesseitig und naturphilosophischpantheistisch und nur sehr weitläufig christlich orientierten Unitarischen Kirche kompatibel war mit seiner fortdauernd betonten christlichen Ausrichtung, mit seiner zeitlebens prägenden lutherischen Herkunft und seinem späten Interesse an der katholischen Kirche.

Wie der Theologe und Literaturwissenschaftler Karl-Josef Kuschel in seinem Aufsatz Lob der Gnade - Lob der Vergänglichkeit. Zum doppelten Ausgang des Werkes von Thomas Mann hervorhebt, wechseln sich in Thomas Manns späteren Romanen, Essays, Ansprachen und Briefen zwei denkerisch unverbundene, wechselnd zum Ausdruck gebrachte Hauptmotive als parallel verlaufende Stränge gegenseitig ab: Zum einen das Festhalten an der Hoffnung auf eine Gnade (wie im Doktor Faustus, der Legende vom Erwäblten und schließlich in der Ansprache an die Hamburger Studenten von 1953), die manchmal apersonal als "Macht" (so in Meine Zeit 1950), manchmal personal verstanden wird (wie in der Anspracbe an die Hamburger Studenten) - zum anderen eine deutliche Betonung des Diesseitigen, des episodenhaften Charakters des Lebens als „Zwischenfall“ in der Einheit des Kosmos; man denke an Felix Krulls Speisewagengespräch mit Professor Kuckuck oder den späten Essay Lob der Vergänglichkeit. Als Schriftsteller spricht Thomas Mann entweder von der Gnade oder vom Lob der Vergänglichkeit, aber nie von beidem gleichzeitig. Und wo ein Hinweis auf die Transzendenz auftaucht, geschieht das ohne weitere kategorial-begriffliche Ausdeutungen. Ferner beschränkt sich jedes Reden Thomas Manns über Christlichkeit, solange der Schriftsteller in Europa lebt, ganz auf sein Werk, ohne den Ansatz eines praktisch kirchlichen Engagements in einer der europäischen Religionsgemeinschaften. Ganz anders im amerikanischen, im kalifornischen Exil. Hier äußert sich Thomas Manns Überzeugung an der Schnittstelle zwischen Humanismus und Religion zum überhaupt ersten Mal über das Schriftstellerische hinaus auch praktisch in seiner aktiven und engagierten Mitwirkung in einer Kirche, im Gemeindeleben und in den Riten von Taufe und Beerdigung. Hier schlägt sich Thomas Manns Überzeugung von der Bedeutung dieser Kirche für ihn in seinen persönlichen, auch schriftlichen Zeugnissen explizit nieder (in Briefen, Tagebuchnotizen, Ansprachen). So erscheinen Humanität und Christlichkeit, trotz allen Unterschieden der wechselnden Gewichtungen, weniger als komplementäre denn als zusammengehörige Größen. Im Kontext von Thomas Manns Beziehung zur Unitarischen Kirche erscheint mir Heinrich Deterings Versuch einer Antwort auf die Frage nach der Vereinbarkeit dieser verwirrenden Zweiheit 
durchaus überzeugend: Es handelt sich für Thomas Mann nicht um ein EntwederOder, sondern um eine offene, universale Synthese.

Persönlich erblicke ich in der Hinwendung Thomas Manns zur Unitarischen Kirche und in meiner durch ihn veranlassten unitarischen Taufe nicht nur einen gewissermaßen stillschweigend erteilten „Auftrag“ an mich und an meine Nachkommen, die Realisierung religiöser Überzeugungen und ethischer Grundwerte in seinem Sinne in irgendeiner Weise fortzusetzen. Die unbewusste unitarische Prägung in meinem Geburtsland USA ist wohl doch stärker gewesen als das im engeren Sinne christliche Gedankengut, mit dem ich später in Berührung kam. Jedenfalls hat sie mir etwas sehr Spezifisches hinterlassen: nämlich die Grundhaltung einer von Toleranz, Dialogbereitschaft und einem demokratisch-gleichberechtigten Pluralismus bestimmten, religionsübergreifenden Sinn- und Werteorientierung, eine daraus erwachsende Ehrfurcht und ein Gefühl der Verantwortung für alles Leben auf unserer Erde und für alles Sein, auch in den verstandesmäßig nicht mehr begreifbaren Dimensionen unseres Kosmos. Diese Grundhaltung habe ich gemeinsam mit Freunden in politischen und kulturellen Projekten praktisch zum Ausdruck zu bringen versucht.

In seiner unitarischen Kanzelrede 1951 formuliert Thomas Mann die angestrebte Synthese von Humanismus und Religion, von Natur und Übernatur, Diesseits und Jenseits, Pantheismus und Christentum, von Schöpfung und Erlösung aus seinem lebenspraktischen Umgang mit Religion und Kirche im amerikanischen Exil heraus:

Today, more urgently, perhaps, than ever before, what is needed, is applied religion, applied Christianity, or, if you prefer, a new, religiously-tainted humanism, aggressively bent on bettering man's status and condition on earth, while, at the same time, honoring, and bowing in reverence to, the secret which lies at the bottom of all human existence, and which must and will never be lifted, - for it is holy.

Diese Sätze sind nicht nur von historischem Interesse, sondern können zum Nachdenken anleiten über den eigenen persönlichen Standort in der Frage einer religiösen, humanen, existenziellen Sinnfindung. 